\title{
Hydrogen-induced unzipping of single-walled carbon nanotubes
}

\author{
Gang Lu \\ Department of Physics, Harvard University, Cambridge, Massachusetts 02138, USA \\ Henry Scudder and Nicholas Kioussis \\ Department of Physics, California State University, Northridge, California 91330-8268, USA
}

(Received 23 July 2003; published 20 November 2003)

\begin{abstract}
The chemisorption of atomic hydrogen on the single-walled armchair and zigzag carbon nanotubes is studied with ab initio calculations. The binding energy of $\mathrm{H}$ adsorption at the exterior of the nanotubes is much greater than that at the interior. We predict that two rows of $\mathrm{H}$ atoms chemisorbed on selective sites exterior to the smaller armchair nanotubes can break the nearest-neighbor $\mathrm{C}-\mathrm{C}$ bonds of the nanotubes through the concerted formation of $\mathrm{C}-\mathrm{H}$ bonds, leading to the unzipping of the nanotube wall. On the other hand, the larger armchair and zigzag nanotubes are stable against unzipping. We provide insights into the underlying electronic mechanism for the $\mathrm{H}$-induced unzipping, lending strong support to the recent experimental observations for the coalescence of single-walled nanotubes in the presence of atomic hydrogen. Interestingly, $\mathrm{H}$ atoms chemisorbed inside the nanotubes do not break the $\mathrm{C}-\mathrm{C}$ bonds.
\end{abstract}

DOI: 10.1103/PhysRevB.68.205416

PACS number(s): 61.48.+c, 61.46.+w, 61.50.Ah, 68.43. $-\mathrm{h}$

Carbon nanotubes have been attracting considerable interest due to their unique electronic and mechanical properties since their discovery about a decade ago. ${ }^{1}$ In particular, there have been intense studies in evaluating the capability of carbon nanotubes as hydrogen storage material for clean energy sources. ${ }^{2,3}$ It was found that hydrogen could be stored in bundles of the single-walled nanotubes up to 5-10 wt $\%$ at pressures less than 1 bar near room temperature. ${ }^{4}$ The mechanism, for the most part, is attributed to physisorption of $\mathrm{H}_{2}$ molecules both inside the tubes and within the interstitial regions among the nanotubes at room or low temperatures. ${ }^{4,5}$ However, recently Liu et al. observed that, after treating carbon nanotubes with $\mathrm{H}_{2}$ gas under high pressure, there was residual $\mathrm{H}_{2}$ during the desorption cycle, ${ }^{3}$ suggesting the presence of chemisorption in the process. On the other hand, chemisorption can also take place during electrochemical storage processes, in which $\mathrm{H}_{2}$ molecules are broken into $\mathrm{H}$ atoms with the application of metal catalysts or electrochemical techniques. ${ }^{6,7}$ On the theoretical side, first-principles calculations have confirmed the dissociative chemisorption of $\mathrm{H}_{2}$ on two adjacent nanotubes in solid phase proceeding with the breaking of the $\mathrm{H}-\mathrm{H}$ bond concerted with the formation of two $\mathrm{C}-\mathrm{H}$ bonds on two adjacent nanotubes. ${ }^{8}$ The chemisorption of atomic $\mathrm{H}$ on single-walled nanotubes has also been observed from selfconsistent-charge-density tight-binding calculations., ${ }^{9,10}$

Recently, Nikolaev and co-workers discovered a remarkable phenomenon; ${ }^{11}$ under atomic $\mathrm{H}$ atmosphere, singlewalled armchair nanotubes annealed up to $1500^{\circ} \mathrm{C}$ coalesce with neighboring tubes, resulting in larger nanotubes with twice and occasionally three times the diameter of the original ones. Based on these observations, the authors proposed a $\mathrm{H}$-activated coalescence mechanism, in which the gasphase $\mathrm{H}$ atoms attack the side of neighboring nanotubes breaking the $\mathrm{C}-\mathrm{C}$ bonds and producing defective sites on the adjacent nanotubes. Once these adjacent defects are formed, the strong thermodynamic force, resulting from the released strain energy in forming larger tubes, drives the two neighboring smaller tubes to join together. Coalescence of clean single-walled nanotubes has also been observed under electron irradiation conditions at elevated temperatures. ${ }^{12}$

In this paper we present a systematic first-principles study of the $\mathrm{H}$-induced unzipping phenomenon (scission of a row of $\mathrm{C}-\mathrm{C}$ bonds along the tube axis) of single-walled nanotubes. The calculations reveal that for the armchair tubes, there is a maximum radius beyond which the tubes cannot be unzipped by $\mathrm{H}$ atoms. More specifically, the smaller $(5,5)$, $(6,6),(7,7),(8,8)$, and $(9,9)$ tubes exhibit the unzipping effect, whereas, the larger $(10,10)$ and $(11,11)$ tubes resist the $\mathrm{H}$-induced unzipping. In contrast, zigzag tubes are more stable and cannot be unzipped by $\mathrm{H}$. We select the $(6,6)$ armchair nanotube as a representative case to demonstrate the generic unzipping mechanism for which a detailed analysis is carried out. Our theoretical calculations lend strong support to the recent experimental observations of $\mathrm{H}$-activated coalescence of armchair nanotubes, ${ }^{11}$ revealing the electronic mechanism responsible for this remarkable phenomenon.

Our ab initio calculations are based on density-functional theory with the CASTEP implementation and ultrasoft pseudopotentials. ${ }^{13}$ The Perdew-Burke-Ernzerhof gradientcorrected functional ${ }^{14}$ was used for the exchange-correlation potential. The energy cutoff for the plane-wave basis was set to be $300 \mathrm{eV}$ yielding a convergence for the total energy better than $1 \mathrm{meV} /$ atom. For the reciprocal-space integration we have used $18 k$ points along the direction corresponding to the nanotube axis using the Monkhorst-Pack scheme. ${ }^{15}$ Results have been obtained for the fully relaxed geometries including all atoms and the lattice constant of the supercell along the tube axis.

The single-walled $(6,6)$ armchair carbon nanotube is modeled with a supercell of dimension $20 \times 20 \times 2.44 \AA^{3}$. This corresponds to two layers of $\mathrm{C}$ atoms perpendicular to the tube axis with 12 atoms per layer. We have studied three different $\mathrm{H}$ coverage, i.e., 1, 2 and $24 \mathrm{H}$ atoms per unit cell. We should point out that due to the periodic boundary con- 
TABLE I. Binding energy (electron volt) per $\mathrm{H}$ atom for chemisorption exterior and interior to the nanotube for one $\mathrm{H}$ atom, a pair of $\mathrm{H}$ atoms on the same and the adjacent layers, as well as the fully hydrogenated case.

\begin{tabular}{lcccc}
\hline \hline & $1 \mathrm{H}$ & $\begin{array}{c}2 \mathrm{H} \text { same } \\
\text { layer }\end{array}$ & $\begin{array}{c}2 \mathrm{H} \text { adjacent } \\
\text { layers }\end{array}$ & $24 \mathrm{H}$ \\
\hline Interior & 0.49 & 0.37 & 0.73 & 0.30 \\
Exterior & 1.77 & 3.00 & 2.52 & 0.84 \\
\hline \hline
\end{tabular}

ditions along the tube axis, 1, 2, and 24 rows of $\mathrm{H}$ atoms are actually being simulated in the calculations. The diameter of the fully relaxed pure nanotube is found to be $8.17 \AA$. The binding energy per $\mathrm{H}$ atom,

$$
E_{b}=(1 / n)\left[E_{\text {tot }}(\text { tube })+E_{\text {tot }}(n \mathrm{H})-E_{\text {tot }}(\text { tube }+n \mathrm{H})\right],
$$

is calculated in terms of the total energy of the pure nanotube $E_{\text {tot }}$ (tube), the total energy of the $n \mathrm{H}(n=1,2,24)$ atoms $E_{\text {tot }}(n \mathrm{H})$, and the total energy of the nanotube with $n \mathrm{H}$ atoms, $E_{\text {tot }}($ tube $+n \mathrm{H})$. Since $E_{\text {tot }}(n \mathrm{H})$ is calculated using the same supercell geometry as in the (tube $+n \mathrm{H})$ system, the spurious adatom-adatom interactions along the tube axis are subtracted. In Table I we list values of the binding energy per $\mathrm{H}$ atom at the interior and exterior of the nanotube for one $\mathrm{H}$ atom, a pair of $\mathrm{H}$ atoms on the same and adjacent layers perpendicular to the nanotube axis, as well as the fully hydrogenated case. The positive value of $E_{b}$ in all cases indicates that the chemisorption is exothermic and hence energetically stable.

First, we consider the $\mathrm{H}$ coverage of one atom per unit cell, with the $\mathrm{H}$ atom adsorbed either at the interior or the exterior of the tube. By placing the $\mathrm{H}$ atom at various initial positions away from the nanotube wall, we find that there is no energy barrier for $\mathrm{H}$ chemisorption at both inside and outside of the tube. Fig. 1(a) shows the relaxed atomic structure with the $\mathrm{H}$ atom adsorbed at the exterior to the tube. We label the nearest-neighbor $\mathrm{C}$ atom to the $\mathrm{H}$ atom by $\mathrm{C}_{1}$, and the nearest-neighbor $\mathrm{C}$ atom to $\mathrm{C}_{1}$ at the same layer by $\mathrm{C}_{2}$. The calculated $\mathrm{C}_{1}-\mathrm{H}$ bond length $(1.11 \AA)$ is the same for the $\mathrm{H}$ atom at either side of the tube wall, and it is close to the corresponding value of $1.10 \AA$ of a $\mathrm{CH}_{4}$ molecule. However, there is a significant difference in the binding energy for $\mathrm{H}$ at the interior $(0.49 \mathrm{eV})$ and the exterior $(1.77 \mathrm{eV})$ of the nanotube wall. This is due to the fact that the chemisorption of $\mathrm{H}$ results in a transition from $s p^{2}$-like bonding in the pure nanotube to $s p^{3}$-like bonding in the presence of $\mathrm{H}$ : and the latter cannot be fully formed upon adsorption to the inner wall because of the unfavorable bonding angle. ${ }^{16}$ The Mulliken analysis of bond population ${ }^{17-19}$ shows that 0.33 electrons is transferred from the $\mathrm{H}$ atom to its nearest-neighbor $\mathrm{C}_{1}$ in agreement with previous calculations ${ }^{20}$ for the fully hydrogenated nanotubes. It is important to note that for the pure nanotube the overlap population between the nearestneighbor $\mathrm{C}$ atoms on the same and two adjacent layers is 0.87 and 2.22 , respectively, indicating a much stronger bonding in the latter case. ${ }^{18}$ This large difference in overlap popu- lation between the two types of nearest-neighbor $\mathrm{C}$ bonds will in turn affect the propensity of $\mathrm{H}$-induced breaking of the $\mathrm{C}-\mathrm{C}$ bonds. The chemisorbed $\mathrm{H}$ results in an increase of the $\mathrm{C}_{1}-\mathrm{C}_{2}$ bond length from $1.42 \AA$ (in the pure nanotube) to $1.53 \AA$, with the latter value being typical for $s p^{3} \mathrm{C}-\mathrm{C}$ bonds. As a result, the $\mathrm{C}_{1}-\mathrm{C}_{2}$ overlap population decreases from 0.87 to 0.63 indicating that the $\mathrm{C}-\mathrm{C}$ bond is weakened. In order to gain more insights into the change of the electronic structure accompanying the $\mathrm{H}$ chemisorption process, we have calculated the angular momentum- and siteprojected density of states (DOS) for some representative cases (Fig. 2). For the pure nanotube, we have confirmed the metallic behavior of the $(6,6)$ armchair nanotube with a low density of states at the Fermi energy $\left(E_{F}\right) .{ }^{1}$ From Fig. 2(a), we find that the $\mathrm{H}$ chemisorption induces the opening of a band gap $(\approx 5 \mathrm{eV})$ for the $\mathrm{C}_{1}$ atom, which is the result of the large charge transfer from the $\mathrm{H}$ atom to $\mathrm{C}_{1}$, filling up the $\mathrm{C}_{1}$ $p$ valence band. On the other hand, it is interesting to note that the $\mathrm{C}_{2}$ atom exhibits a large narrow peak across $E_{F}$, which overlaps with the bonding states of the $\mathrm{H}$ atom. Figure 3(a) shows the valence charge density contour plot in the presence of a single $\mathrm{H}$ for a plane containing the $\mathrm{C}$ atoms on the same layer. One can observe the directional $s p^{2}$-like covalent bonding between the $\mathrm{C}$ atoms, the $s p^{3}$-like bonding between $\mathrm{C}_{1}$ and $\mathrm{H}$, and the weakening of the $\mathrm{C}_{1}-\mathrm{C}_{2} s p^{2}$ bond.

The result of the $\mathrm{H}$-induced weakening of the $\mathrm{C}-\mathrm{C}$ bond invites an interesting question: Could the chemisorption of another $\mathrm{H}$ atom on the nearby $\mathrm{C}$ atom lead to the formation of two $\mathrm{H}-\mathrm{C}$ bonds, which will in turn further decrease or even break the $\mathrm{C}_{1}-\mathrm{C}_{2}$ bond resulting in the unzipping of the entire nanotube? Thus, next we consider the case of a pair of $\mathrm{H}$ atoms chemisorbed on the $\mathrm{C}_{1}$ and $\mathrm{C}_{2}$ atoms in the exterior and the interior to the tube. We have examined two different adsorption sites for the second $\mathrm{H}$ atom, either at the same or the adjacent $\mathrm{C}$ layer. First, we present results with the interatomic axis between the $\mathrm{H}$ atoms parallel to the $\mathrm{C}_{1}-\mathrm{C}_{2}$ bond. The binding energy per $\mathrm{H}$ for this case is 0.37 $\mathrm{eV}$ (inside the tube) and $3.0 \mathrm{eV}$ (outside the tube), respectively. As in the single $\mathrm{H}$ case, the pair of $\mathrm{H}$ atoms prefers to be chemisorbed at the exterior of the nanotube. Interestingly, the binding energy per $\mathrm{H}$ atom for the pair outside the nanotube has dramatically increased as compared to the corresponding value for the single $\mathrm{H}$ case, while the charge transfer from each $\mathrm{H}$ to its nearest-neighbor $\mathrm{C}$ remains about the same ( 0.30 electrons $)$ as in the one $\mathrm{H}$ case. This charge transfer leads to an ioniclike repulsion between the $\mathrm{H}$ atoms and between $\mathrm{C}_{1}$ and $\mathrm{C}_{2}$. Figure $1(\mathrm{~b})$ shows the relaxed atomic structure for the case of two $\mathrm{H}$ atoms adsorbed at the exterior of the tube on the same layer. The most remarkable feature in this figure is the breaking of the nearest-neighbor $\mathrm{C}_{1}-\mathrm{C}_{2}$ bond. The equilibrium $\mathrm{H}-\mathrm{C}, \mathrm{C}_{1}-\mathrm{C}_{2}$, and $\mathrm{H}-\mathrm{H}$ bond lengths are $1.09 \AA, 4.07 \AA$, and $2.94 \AA$, respectively. Due to the periodic boundary conditions along the tube axis, the result implies scission of the nanotube wall along the tube axis, i.e., the unzipping effect necessary for the experimentally observed coalescence phenomenon. For the same reason, the unzipped tube cannot fully extend or flatten out due to the repulsion of the nanotubes in the neighboring unit 

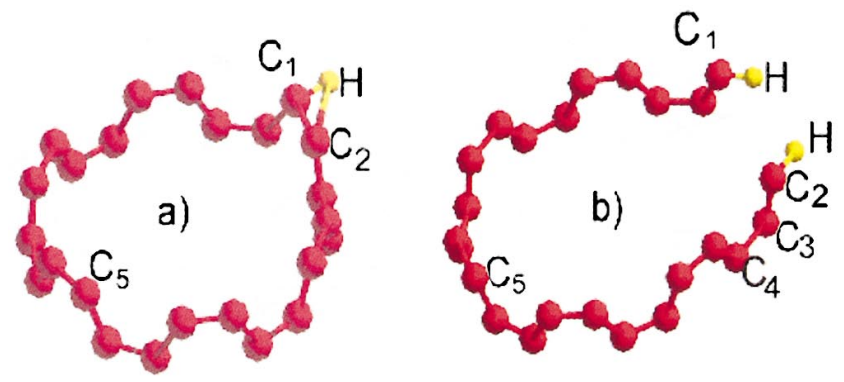

cells. The overlap population of $\mathrm{C}_{2}-\mathrm{H}$ is 0.71 , which is as large as that for the nearest-neighbor $\mathrm{C}-\mathrm{C}$ bond at the same layer in the pure nanotube. Figure 2(b) shows the DOS for one of the hydrogenated $\mathrm{C}$ atoms, $\mathrm{C}_{2}$ [see also Fig. 1(b)] and its nearest neighbor $\mathrm{C}_{3}$. The DOS for $\mathrm{C}_{2}$ is similar to that of $\mathrm{C}_{1}$ in Fig. 2(a). Note the narrowing of the $\mathrm{H} s$ band, which exhibits bonding and antibonding states separated by an energy gap of about $5 \mathrm{eV}$, indicates that the $\mathrm{H}$ atom is stabilized due to the hybridization between the $s$ states of $\mathrm{H}$ and the $p$ states from the nearby $\mathrm{C}_{2}$ atom. From Fig. 3(b), one can see the $s p^{3}$-like bonding between the $\mathrm{C}$ and the $\mathrm{H}$ atoms, and the breaking of the $\mathrm{C}_{1}-\mathrm{C}_{2}$ bond.

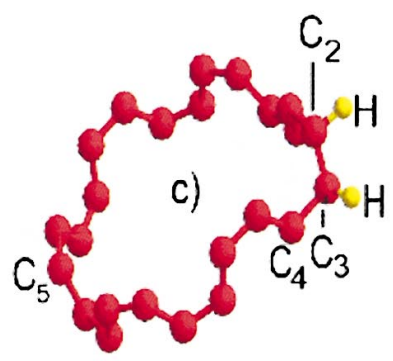

FIG. 1. (Color) Relaxed atomic structures for chemisorption of one $\mathrm{H}$ atom (a), and a pair of $\mathrm{H}$ atoms on the same layer (b) and on the adjacent layers (c) of the $(6,6)$ single-walled armchair nanotube.

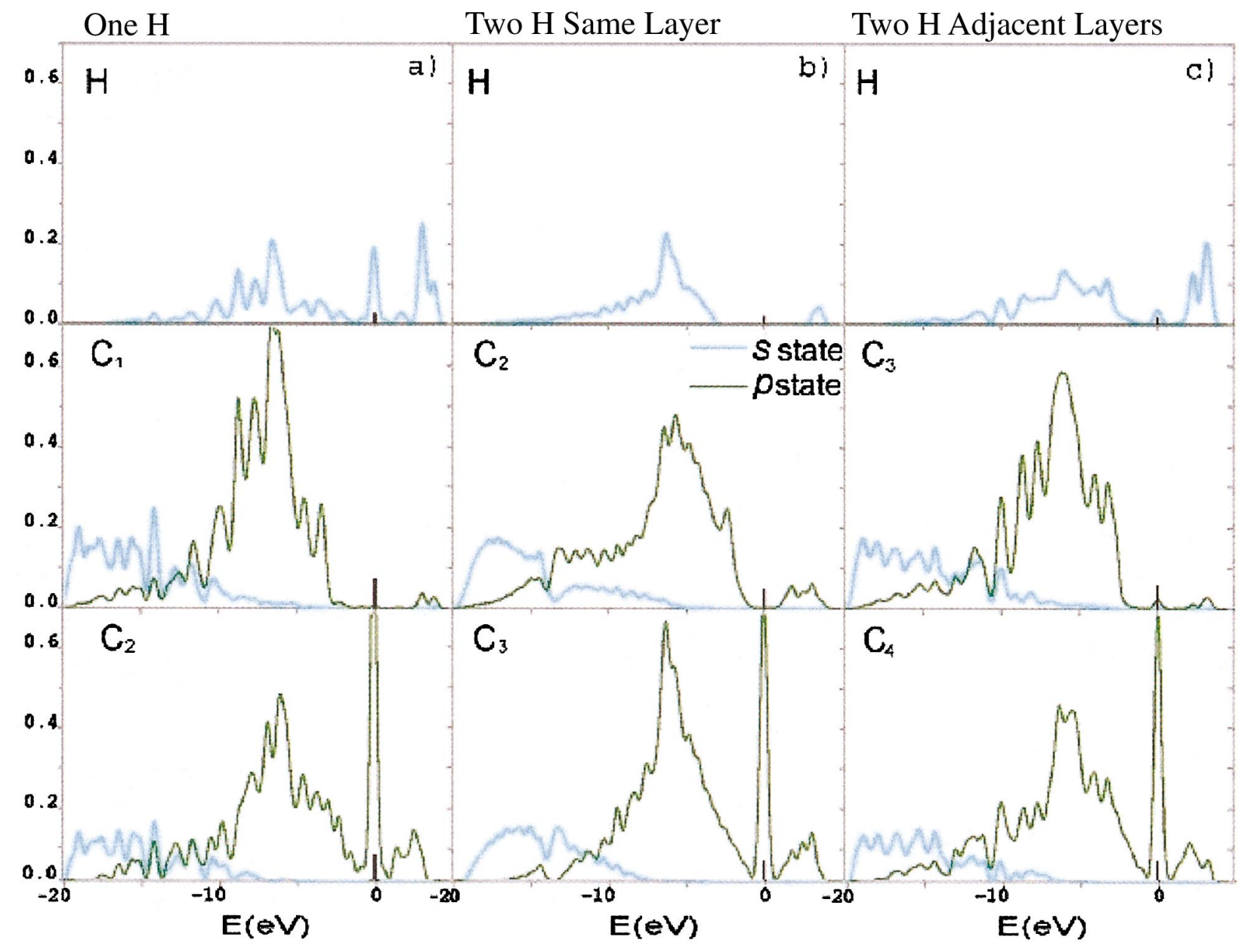

FIG. 2. (Color) The angular momentum and site projected DOS for the adsorption of one $\mathrm{H}$ atom (a), and a pair of $\mathrm{H}$ atoms on the same layer (b) and on the adjacent layers (c), exterior to the nanotube. The labeling of the various atoms is the same as that used in Figs. 1(a)-1(c), respectively. 


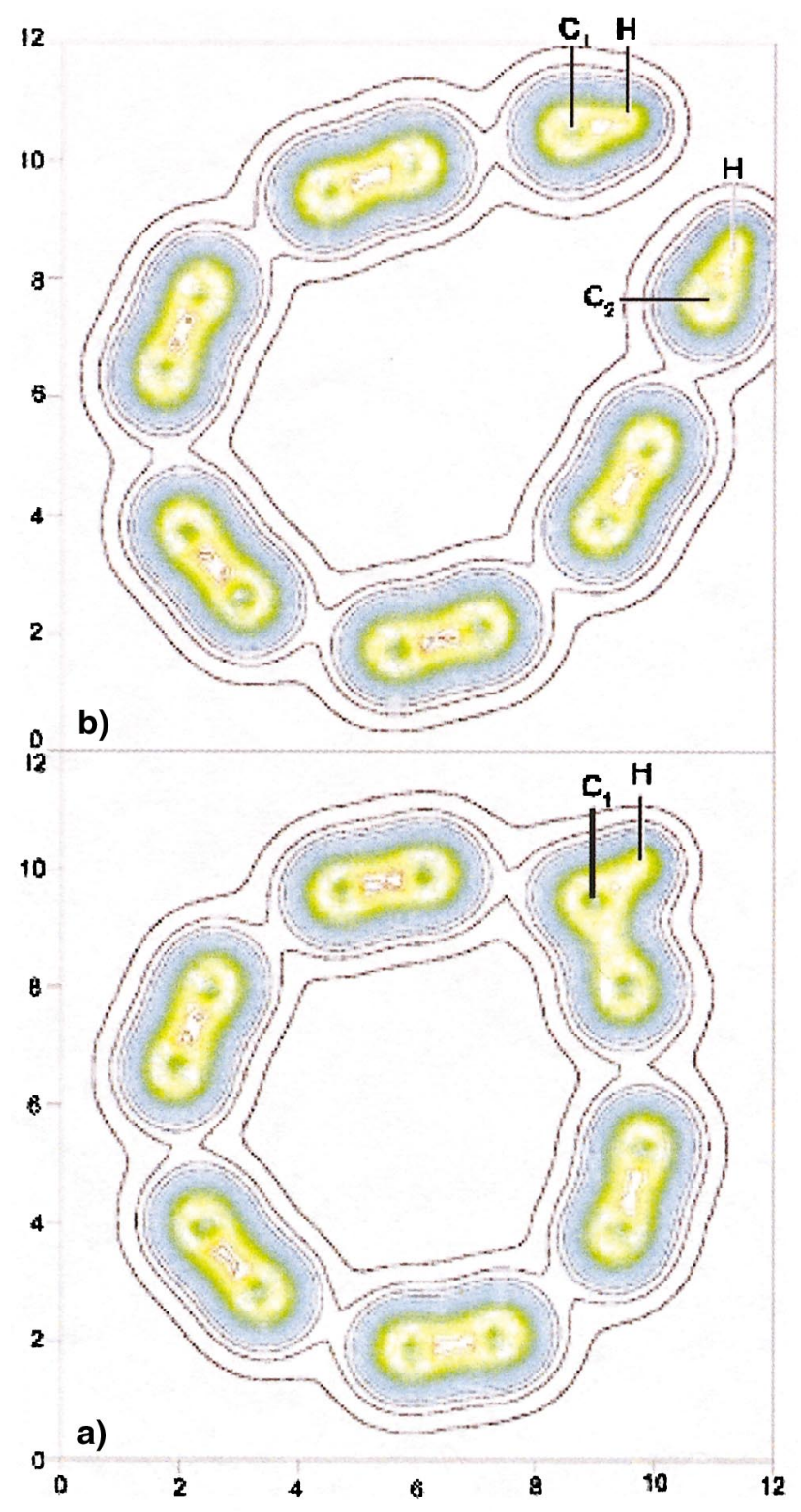

FIG. 3. (Color) Valence charge density contour plot on a plane perpendicular to the nanotube axis passing through the $\mathrm{C}$ atoms, for adsorption of one $\mathrm{H}$ atom (a), and two $\mathrm{H}$ atoms on the same layer (b).

of 2.2 in the pure nanotube. By employing a larger supercell (four layers per cell), we find essentially the same results, i.e., the $\mathrm{C}-\mathrm{C}$ bond remains unbroken in the presence of $\mathrm{H}$ atoms. This result can be understood from the fact that the nearest-neighbor $\mathrm{C}-\mathrm{C}$ bonds on adjacent layers are much stronger than those on the same layer, as alluded earlier.

Interestingly, the $\mathrm{C}-\mathrm{C}$ bond cannot break when the two $\mathrm{H}$ atoms chemisorb inside the nanotube regardless of the adsorption site. The $\mathrm{C}-\mathrm{C}$ bond deforms nevertheless, with the bond length increasing from $1.42 \AA$ (pure tube) to 1.52 $\AA$. This again originates from the reduced propensity for $s p^{3}$ $\mathrm{H}-\mathrm{C}$ bonding at the interior of the nanotube. ${ }^{16}$ Overall, the internal surface of the nanotube is found to be less reactive than the external surface, in agreement with the general consensus. ${ }^{21,22}$ Finally, our calculations for the fully endohydrogenated and exo hydrogenated nanotube find no breaking of the $\mathrm{C}-\mathrm{C}$ bonds and a lower binding energy per $\mathrm{H}$ atom of $0.30 \mathrm{eV}$ and $0.84 \mathrm{eV}$, respectively. We have also carried out calculations for a $\mathrm{H}_{2}$ molecule approaching the nanotube wall, both from the exterior and the interior, with the $\mathrm{H}-\mathrm{H}$ bond perpendicular to the nanotube wall. In agreement with previous calculations, ${ }^{8}$ we find that no chemisorption occurs and the $\mathrm{H}_{2}$ molecule remains intact.

In order to clarify the effect of the nanotube radius on the propensity of unzipping, we have carried out similar calculations for the $(5,5),(7,7),(8,8),(9,9),(10,10)$, and $(11,11)$ armchair tubes. In all cases the two $\mathrm{H}$ atoms are chemisorbed outside the tube on two nearest-neighbor $\mathrm{C}$ atoms at the same layer, i.e., same configuration used for the $(6,6)$ tube. We find that all the four smaller tubes exhibit the same $\mathrm{H}$-induced unzipping, while the larger $(10,10)$ and $(11,11)$ tubes remain intact, i.e., no $\mathrm{C}-\mathrm{C}$ bond is broken. These results are consistent with the fact that the $\mathrm{C}-\mathrm{C}$ bonds in smaller radii nanotubes are under greater strain (especially those perpendicular to the tube axis ${ }^{23,24}$ ), therefore these $\mathrm{C}-\mathrm{C}$ bonds are more susceptible to the H-induced unzipping. In order to have a more complete picture of the unzipping phenomenon, we have performed additional calculations for the $(7,0),(9,0)$, and $(10,0)$ zigzag tubes with two $\mathrm{H}$ atoms chemisorbed exterior to the tubes. In the zigzag tubes there are two inequivalent nearest-neighbor $\mathrm{C}-\mathrm{C}$ bonds: one parallel and the other zigzag to the tube axis. Since none of these bonds in a zigzag tube are strained as much as those on the same layer (perpendicular to the tube axis) in an armchair tube, one would expect that the zigzag tubes are more stable against the $\mathrm{H}$-induced unzipping. Indeed, our calculations confirm that the zigzag tubes are more stable and that their $\mathrm{C}-\mathrm{C}$ bonds remain intact. Thus, our results indicate that the $\mathrm{C}-\mathrm{C}$ bonds that are perpendicular (parallel) to the tube axis are the most (least) strained, and that the strain of the zigzag $\mathrm{C}-\mathrm{C}$ bonds lies in between the two extreme cases. This general rule is also consistent with the finding that the $\mathrm{C}-\mathrm{C}$ bonds at two adjacent layers of the $(6,6)$ tube are stronger than those at the same layer. Finally, our results suggest that the metallic/semiconducting nature of a nanotube is not the deciding factor for the unzipping phenomenon. Some of the metallic tubes, such as the smaller armchair tubes can be unzipped, while the other metallic tubes, such as the larger armchair tubes and the $(9,0)$ zigzag tube cannot be unzipped. More calculations are needed to ascertain if all semiconducting tubes are stable against unzipping. Also, since our $a b$ initio calculations are carried out at zero temperature, one should be cautious to compare the theoretical predictions with the finite-temperature experimental results.

Recently, similar density-functional calculations were performed by Arellano et al. to study the interaction of molecular and atomic $\mathrm{H}$ with the $(5,5)$ and $(6,6)$ nanotubes. ${ }^{25} \mathrm{Al}$ though these authors observed the $\mathrm{H}$-induced unzipping in the $(6,6)$ tube, consistent with our finding, they found that the $(5,5)$ tube is on the verge of being unzipped. Their result for the $(5,5)$ tube is inconsistent with ours and other 
calculations $^{23,24}$ which indicate that the $\mathrm{C}-\mathrm{C}$ bonds in smaller armchair tubes are weaker, and hence are more susceptible to unzipping.

In conclusion, we have studied the $\mathrm{H}$-induced unzipping of single-walled nanotubes. We find that $\mathrm{H}$ atoms bind strongly to the nanotubes through $s p^{3}$ bonding, with the binding energy at the exterior of the tube being much greater than that at the interior. We predict that for the smaller armchair nanotubes, a pair of $\mathrm{H}$ atoms chemisorbed on the two nearest-neighbor $\mathrm{C}$ atoms of the same layer exterior to the tubes catalyze the breaking of the $\mathrm{C}-\mathrm{C}$ bond and lead to the unzipping of the nanotubes. On the other hand, the larger armchair tubes and zigzag nanotubes are stable against unzipping. This $\mathrm{H}$-induced unzipping mechanism lends strong support to the recent experimental observations for the coalescence of smaller nanotubes into larger radius nanotubes in the presence of atomic H. For all the nanotubes under study, we find that there is no $\mathrm{H}$-induced unzipping when $\mathrm{H}$ atoms are chemisorbed at the interior of the tubes.

We acknowledge support from the U.S. Army under Grant Nos. DAAD19-00-1-0049 and DAAG55-97-1-0247.
${ }^{1}$ M.S. Dresselhaus, G. Dresselhaus, and P.C. Eklund, Science of Fullerenes and Carbon Nanotubes (Academic, San Diego, 1996); R. Saito, G. Dresselhaus, and M.S. Dresselhaus, Physical Properties of Carbon Nanotubes (Imperial College Press, London, 1998).

${ }^{2}$ P. Chen, X. Wu, J. Lin, and K.I. Tan, Science 285, 91 (1999).

${ }^{3}$ C. Liu, Y.Y. Fan, M. Liu, H. Liu, H.T. Cong, H.M. Cheng, and M.S. Dresselhaus, Science 286, 1127 (1999).

${ }^{4}$ A.C. Dillon, K.M. Jones, T.A. Bekkedahl, C.H. Kiang, D.S.B. Bethune, and M.J. Heben, Nature (London) 386, 377 (1997).

${ }^{5}$ Y. Ye, C.C. Ahn, C. Witham, B. Fultz, J. Liu, A.G. Rindzler, C. Colbert, K.A. Smith, amd R.E. Smalley, Appl. Phys. Lett. 74, 2307 (1999).

${ }^{6}$ R.E. Haufler et al., J. Phys. Chem. 94, 8634 (1990).

${ }^{7}$ L. Becker, T.P. Evans, and J.L. Bada, J. Org. Chem. 58, 7630 (1993).

${ }^{8}$ Siu-Pang Chan, G. Chen, X.G. Gong, and Z.F. Liu, Phys. Rev. Lett. 87, 205502 (2001).

${ }^{9}$ S.M. Lee and Y.H. Lee, Appl. Phys. Lett. 76, 2877 (2000).

${ }^{10}$ S.M. Lee, K.S. Park, Y.C. Choi, Y.S. Park, J.M. Bok, D.J. Bae, K.S. Nahm, Y.G. Choi, S.C. Yu, N. Kim, T. Frauenheim, and Y.H. Lee, Synth. Met. 113, 209 (2000).

${ }^{11}$ P. Nikolaev, A. Thess, A.G. Rinzler, D.T. Colbert, and R. Smalley, Chem. Phys. Lett. 266, 422 (1997).
${ }^{12}$ M. Terrones, H. Terrones, F. Banhart, J.-C. Charlier, and P.M. Ajayan, Science 288, 1226 (2000).

${ }^{13}$ D. Vanderbilt, Phys. Rev. B 41, 7892 (1990).

${ }^{14}$ J.P. Perdew, K. Burke, and M. Ernzerhof, Phys. Rev. Lett. 77, 3865 (1996)

${ }^{15}$ H.J. Monkhorst and J.D. Pack, Phys. Rev. B 13, 5188 (1976).

${ }^{16}$ K. Tada, S. Furuya, and K. Watanabe, Phys. Rev. B 63, 155405 (2001).

${ }^{17}$ R.S. Mulliken, J. Chem. Phys. 23, 1833 (1955).

${ }^{18}$ D. Sanchez-Portal, E. Artacho, and J.M. Soler, J. Phys.: Condens. Matter 8, 3859 (1996).

${ }^{19}$ M.D. Segall, C.J. Pickard, R. Shah, and M.C. Payne, Mol. Phys. 89, 571 (1996).

${ }^{20}$ T. Yildirim, O. Gülseren, and S. Ciraci, Phys. Rev. B 64, 075404 (2001).

${ }^{21}$ R.C. Haddon et al., Nature (London) 350, 320 (1991).

${ }^{22}$ S. Park, D. Srivastava, and K. Cho, Nanotechnology 12, 245 (2001).

${ }^{23}$ X. Blase, L.X. Benedict, E.L. Shirley, and S.G. Louie, Phys. Rev. Lett. 72, 1878 (1994).

${ }^{24}$ D. Sanchez-Portal, E. Artacho, and J.M. Soler, A. Rubio, and P. Ordejon, Phys. Rev. B 59, 12678 (1999).

${ }^{25}$ J.S. Arellano, L.M. Molina, A. Rubio, M.J. Lopez, and A.J. Alonso, J. Chem. Phys. 117, 2281 (2002). 